\title{
The performance and operational experience of ATLAS SemiConductor Tracker in Run-2 at LHC
}

\author{
Sergio Grancagnolo ${ }^{a, *}$ on behalf of the ATLAS SCT Group \\ ${ }^{a}$ Humboldt Universität zu Berlin, \\ Institut für Physik, Newtonstraße 15, \\ 12489 Berlin, Germany \\ E-mail: sergio.grancagnolo@cern.ch
}

The performance of ATLAS SemiConductor Tracker in Run-2 at Large Hadron Collider has been reviewed during the current long shutdown. The LHC successfully completed its Run-2 operation (2015-2018) with a total integrated delivered luminosity of $156 \mathrm{fb}^{-1}$ at the centre-of-mass $p p$ collision energy of $13 \mathrm{TeV}$. The LHC high performance provides a good opportunity for physics analysis. The instantaneous luminosity and pileup conditions were far in excess of what the SCT was originally designed to meet. This document will summarise the operational experience and performance of the SCT during Run-2, with a focus on the impact and mitigation of radiation damage effects, which were observed in Run-2.

\footnotetext{
*** The European Physical Society Conference on High Energy Physics (EPS-HEP2021), *** *** 26-30 July $2021 * * *$

*** Online conference, jointly organized by Universität Hamburg and the research center DESY ***
}

\footnotetext{
* Speaker
} 


\section{Introduction}

During the Run-2 data-taking period, the Large Hadron Collider (LHC) reached twice its design luminosity, delivering $156 \mathrm{fb}^{-1}$ at the $p p$ collision energy of $13 \mathrm{TeV}$, with a peak luminosity of $2 \times 10^{34} \mathrm{~cm}^{-2} \mathrm{~s}^{-1}$. Such exceptional performance came with higher pileup conditions, up to $\sim 60$ interactions per bunch crossing (instead of an expected average pileup of 23). The ATLAS [1] SemiConductor Tracker (SCT), designed for $0.2-0.5 \%$ hit occupancy, was challenged with hit occupancy levels up to $\sim 2 \%$, reaching bandwidth limitations. The improvements to transmission rates and data quality are summarised in the following sections. [2]

\section{Data Acquisition (DAQ)}

Data from the modules are processed by Read Out Drivers (RODs) that format data and send them via optical S-links. If the data volume and rate is too high, the RODs will impose a busy signal to throttle the data rate, giving rise to dead time. Several improvements were then put in place. The number of optical S-links and RODs was expanded from 90 to 128, thereby reducing the volume of data transmitted and processed by the S-links and RODs, respectively. Furthermore, moduleassignment to RODs were rearranged, to get a flatter occupancy distribution across S-links. The Front-End (FE) chip samples three consecutive bunch crossings, centered on the triggered bunch crossing. Contributions to the signal from the previous bunch crossing were vetoed at the chip level by imposing a " $01 \mathrm{X}$ " requirement on the three consecutive samplings, resulting in reduced hit occupancy and reduced noise. An improved data compression by the ROD was implemented: clusters up to 16 strips are packed into a single 16-bit word, to achieve a $\sim 25 \%$ data size reduction.

If high pileup in combination with high trigger rate causes the transmission of $\mathrm{FE}$ data to exceed bandwidth limitations, the FE chips will drop hit information and flag errors (Figure 1). These chips can be masked during operations, and recovered on the fly when pileup conditions

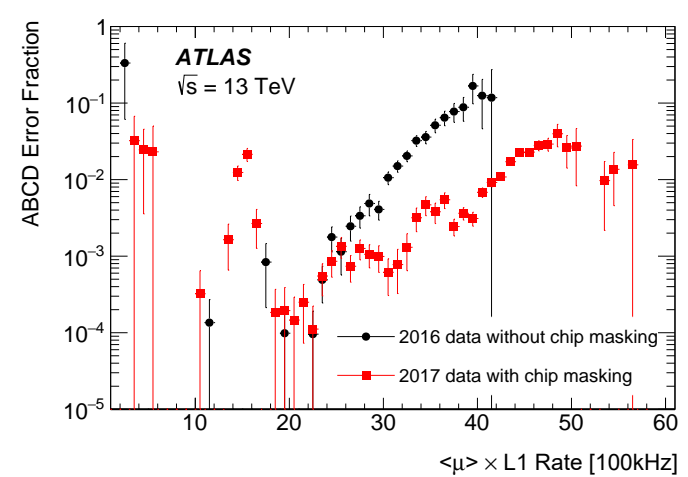

Figure 1: Chip error fraction as a function of pileup times the level-1 accept rate. Even at higher pileup, errors are fewer when chip masking is implemented. [2]

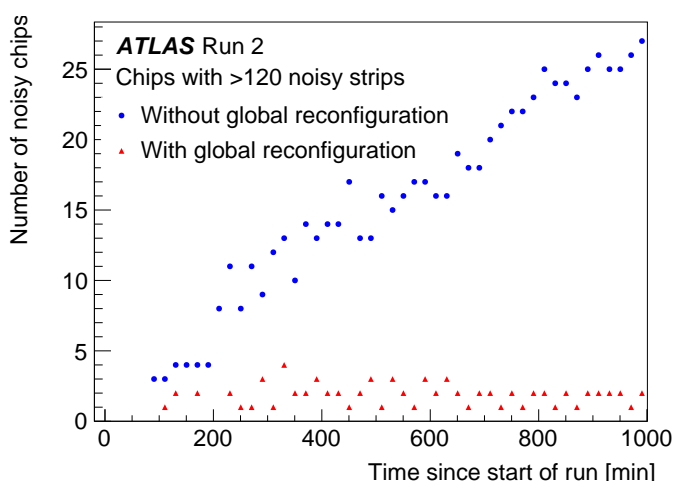

Figure 2: Number of noisy chips since start of run. Global DAQ reconfiguration keeps their number low, during data-taking. [2]

improve. Single Event Effects include module desynchronization (when particles passing through the opt-electronics result in missed or fake triggers) and noisy or quiet modules (when particles 
passing through the FE chips cause bit-flips in the threshold registers). Desynchronized modules can be automatically detected within 20-30 seconds and recovered by reconfiguration. A global DAQ reconfiguration takes $1.2 \mathrm{~s}$ and, for negligible data loss, it is used regularly during data taking, to recover quiet or noisy modules (Figure 2). During Run-2, the dead-time was $<0.1 \%$ on average.

\section{Performance}

The stability of the cluster width (number of contiguous strips with hits) is important for tracking. The cluster width distribution is estimated using good quality tracks (Figure 3). The mean cluster width as a function of a particle incident angle gives a good estimate of the Lorentz angle (Figure 4). Important for barrel modules, where electric and magnetic fields are perpendicular, the Lorentz angle is the deviation of the drift charge direction from the electric field vector, due to the magnetic field. There is a trade off between better charge collection at higher high voltage (HV), increasing the cluster width, and faster charge collection, which decreases the cluster width suppressing transverse charge diffusion.

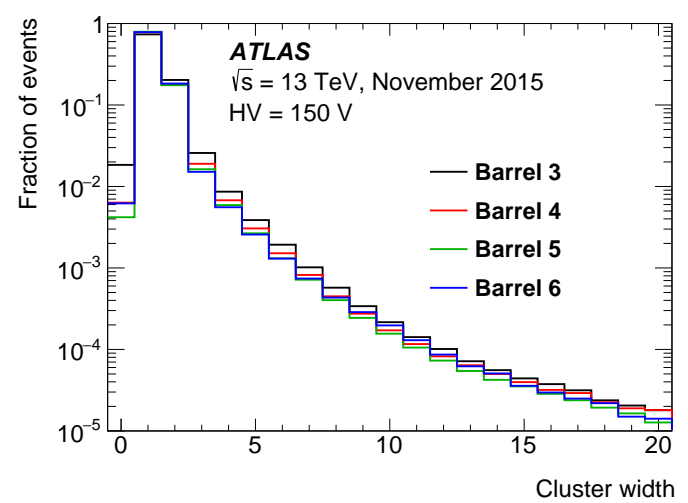

Figure 3: Cluster width distribution in the barrel. It peaks at 1 , has a long tail, and a mean width of $\sim$ 1.2. Clusters with zero width come from tracks with a hole in the corresponding barrel layer. [2]

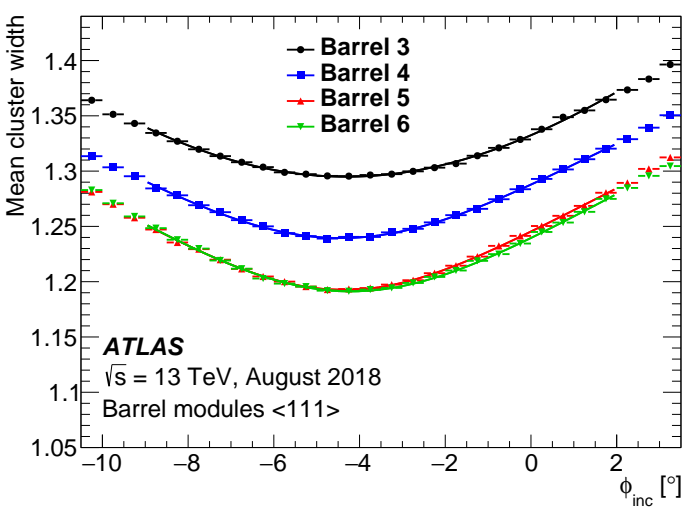

Figure 4: Cluster width vs incident angle. If a particle incident angle equals the Lorentz angle, all induced charge drifts along the particle direction, giving the minimum cluster width. [2]

The hit efficiency is defined using the number of clusters or holes associated to each track: $\epsilon=N_{\text {cluster }} /\left(N_{\text {cluster }}+N_{\text {hole }}\right)$ and estimated using events with less than 500 good-quality tracks. Due to the " $01 \mathrm{X}$ " read mode: a real hit is lost if one is already present in the preceding bunch (1\% hit loss). A redundant design (typically 8 SCT clusters per track) allows to have a negligible impact on tracking. The first bunch crossing in a train is not affected (Figure 5), giving 99\% as intrinsic hit efficiency.

\section{Radiation Damage}

After receiving radiation, performance is distorted due to creation of additional states within the semiconductor band gap, modifying e.g. the full depletion voltage, leakage current, probability of charge trapping. Predictions from simulations agree with measurements from dedicated radiation 


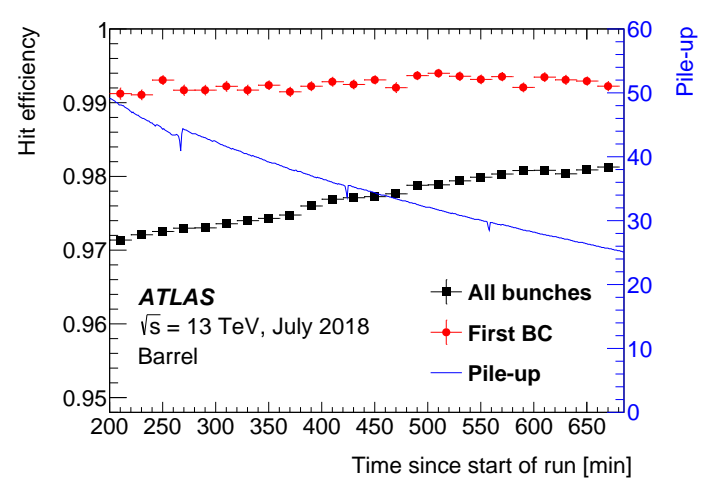

Figure 5: Hit efficiency during a data taking run. The intrinsic hit efficiency remains nearly constant, while it increases as pileup decreases. [2]

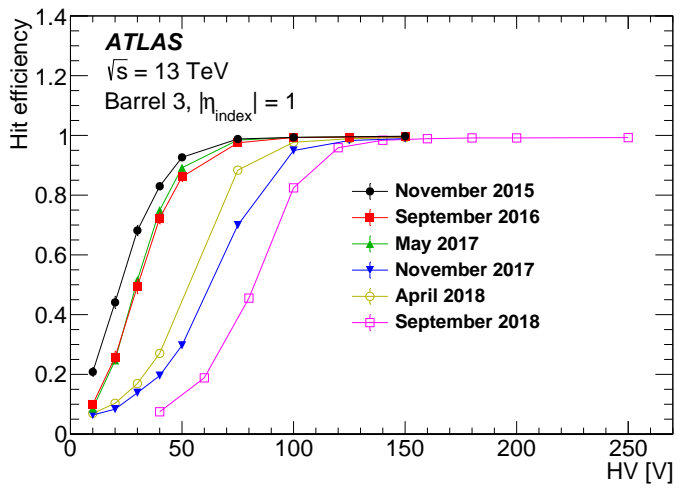

Figure 6: Hit efficiency as a function of operational HV. Irradiated sensors need higher HV to be fully depleted and reach hit efficiency plateau. [2]

monitoring systems: the total fluence amounts to $5.6 \times 10^{13}$ thermal neutrons ( $1 \mathrm{MeV}$ equivalent). The hit efficiency is affected by the radiation damage in the sensors: as the number of donors decreases, the number of acceptors increases. As expected around the second half of Run-2, the sensors inverted from $n$-bulk into $p$-bulk material. After type inversion, full efficiency is reached at an increased HV (Figure 6). As the depletion voltage increased, the operating voltage had to be increased in 2018 , from $150 \mathrm{~V}$ to $200-250 \mathrm{~V}$, to compensate a $0.5 \%$ hit efficiency drop.

Leakage Current is caused by thermally generated electron-hole pairs. It is highly dependent on sensor temperature and is proportional to the depleted volume. If too large, it could cause thermal runaway due to increased power dissipation in the sensors, forcing to lower $\mathrm{HV}$, hence reducing the hit efficiency. Over the course of Run-2, it increased by more than one order of magnitude. This was in agreement with predictions, within $30 \%$ uncertainty. Normalised leakage currents per unit volume for all barrel modules are consistent within $3 \%$. During each winter shutdown it reduced by 20-30\% due to annealing. The noise levels increased by 10-20\%, at the level of $0.32 \mathrm{fC}$ at most, which was still sufficiently low compared to the threshold of $1 \mathrm{fC}$.

\section{Conclusions}

SCT operated stably throughout Run-2, with several new improvements: additional RODs, more aggressive data compression, automatic recovery mechanisms for modules and RODs and reduced calibration time. Leakage current and full depletion voltage were accurately monitored, though not all results are listed here. The SCT achieved a good data quality efficiency of $99.85 \%$. It was available for $99.9 \%$ of integrated luminosity, had a low noise occupancy kept below $5 \times 10^{-4}$ and a high hit efficiency on the order of $\sim 99 \%$. Safe and stable operation is expected until the end of Run-3, with an integrated luminosity of $\sim 200 \mathrm{fb}^{-1}$ at similar pileup levels.

\section{References}

[1] ATLAS Collaboration, 2008 JINST 3 S08003

[2] ATLAS Collaboration, arXiv:2109.02591 